\title{
A new analytical solution for testing debris avalanche numerical models
}

\author{
Sudi Mungkasi ${ }^{1} \quad$ Stephen G. Roberts ${ }^{2}$
}

(Received 14 December 2010; revised 29 June 2011)

\begin{abstract}
An analytical solution to a debris avalanche problem in the one dimensional topography-linked coordinate system was found by Mangeney, Heinrich, and Roche [Pure Appl. Geophys., 157:1081-1096, 2000]. We derive an analytical solution to a debris avalanche problem in the standard Cartesian coordinate system. Characteristics and a transformation technique obtain the analytical solution. This analytical solution is used to test finite volume methods with reconstruction of the conserved quantities based on: either stage, height, and velocity; or stage, height, and momentum. Numerical tests show that the finite volume method with reconstruction based on stage, height, and momentum is slightly more accurate in solving the debris avalanche problem.
\end{abstract}

http://anziamj. austms.org.au/ojs/index.php/ANZIAMJ/article/view/3785 gives this article, (c) Austral. Mathematical Soc. 2011. Published July 12, 2011; amended July 13, 2011. ISSN 1446-8735. (Print two pages per sheet of paper.) Copies of this article must not be made otherwise available on the internet; instead link directly to this URL for this article. 


\section{Contents}

1 Introduction

C350

2 Saint-Venant models

C351

3 Existing solutions

C353

4 A new solution

C354

5 Numerical tests

C357

6 Conclusions

C360

References

C361

\section{Introduction}

We present an analytical solution to a debris avalanche problem (model) based on the Saint-Venant approach. Using the Saint-Venant approach implies that debris is treated as a depth averaged fluid. We use this analytical solution as a benchmark to assess two finite volume methods applied to the model. The considered problem is a simplification of real world scenarios on the avalanches of debris, such as snow, sands, rocks, as well as landslides. In other words, our work can be applied to these real world scenarios.

Three problems are considered. The first is the dam break problem in the standard Cartesian coordinate system having initial condition shown in Figure 1(a). This problem was solved by Ritter [8]. The second is the debris avalanche problem in the topography-linked coordinate system having initial condition illustrated in Figure 1(b). Mangeney et al. [5] proposed a solution to this problem. The third is the debris avalanche problem in the standard 


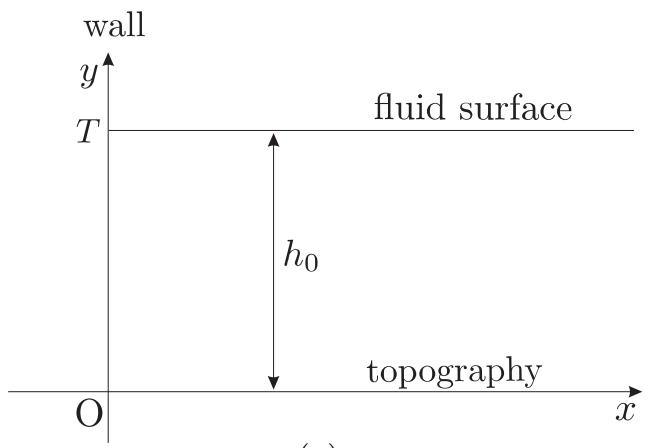

(a)

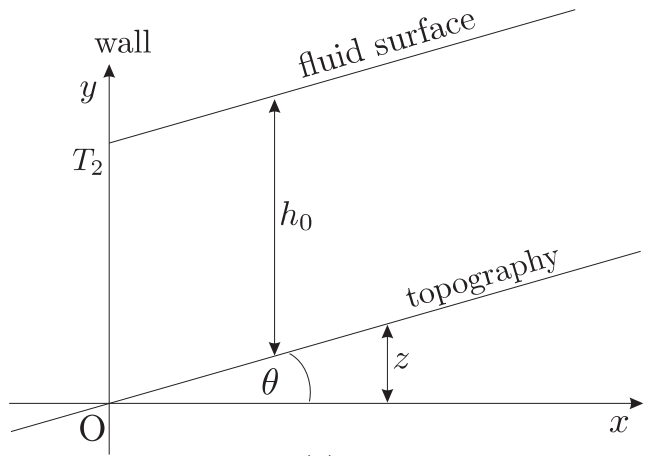

(c)

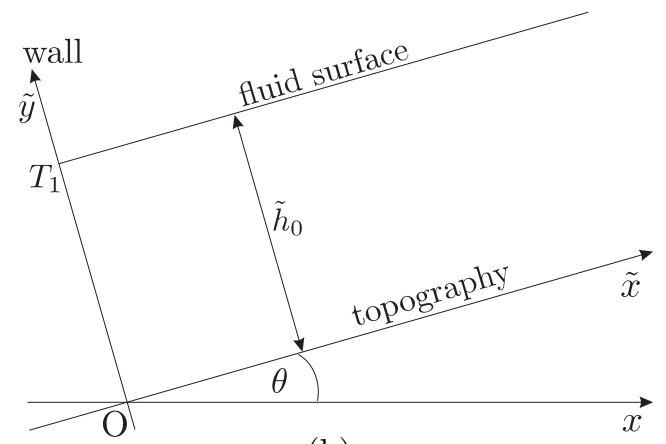

(b)

Figure 1: Initial conditions of three considered problems: (a) a dam break problem in the standard Cartesian coordinate system; (b) a debris avalanche problem in the topography-linked coordinate system; (c) a debris avalanche problem in the standard Cartesian coordinate system.

Cartesian coordinate system having initial condition shown in Figure 1(c). This third problem is the one we solve in this article.

\section{Saint-Venant models}

We review the Saint-Venant model in the standard Cartesian coordinate system and the Saint-Venant model in the topography-linked coordinate system.

In the standard Cartesian coordinate system, the mass and momentum 
differential equations governing the fluid motion are

$$
\begin{aligned}
& h_{t}+(h u)_{x}=0 \\
& (h u)_{t}+\left(h u^{2}+\frac{1}{2} g h^{2}\right)_{x}=-g h z_{x}+h F .
\end{aligned}
$$

These two equations are called the Saint-Venant model in the standard Cartesian coordinate system. Here, $x$ represents the one dimensional spatial variable, $t$ represents the time variable, $u=u(x, t)$ denotes the fluid velocity, $h=h(x, t)$ denotes the fluid height (depth), $z=z(x)$ is the topography (bed), $g$ is the acceleration due to the gravity, and $F$ is a factor representing a Coulomb-type friction. Note that we consider $z_{x}=\tan \theta$, where $\theta$ is the angle between the topography and the horizontal line; $\tan \theta$ is called the bed slope, while $\theta$ is called the bed angle. Equations (1) and (2) can be solved using the characteristic relations [9]

$$
C_{+}: \frac{d x}{d t}=u+c, \quad C_{-}: \frac{d x}{d t}=u-c,
$$

in which the Riemann invariants $k_{ \pm}$are

$$
\begin{aligned}
& u+2 c-m t=k_{+}=\text {constant along each curve } C_{+}, \\
& u-2 c-m t=k_{-}=\text {constant along each curve } C_{-},
\end{aligned}
$$

where $c=\sqrt{g h}$ and $m=-g \tan \theta+F$.

The Saint-Venant model in the topography-linked coordinate system is

$$
\begin{aligned}
& \tilde{h}_{t}+(\tilde{h} \tilde{u})_{\tilde{x}}=0, \\
& (\tilde{h} \tilde{u})_{t}+\left(\tilde{h} \tilde{u}^{2}+\frac{1}{2} g \tilde{h}^{2} \cos \theta\right)_{\tilde{x}}=-g \tilde{h} \sin \theta+\tilde{h} \tilde{F} .
\end{aligned}
$$

We use a tilde notation to identify variables in the topography-linked coordinate system. Equations (6) and (7) can be solved using characteristic relations

$$
\tilde{C}_{+}: \frac{d \tilde{x}}{d t}=\tilde{u}+\tilde{c}, \quad \tilde{C}_{-}: \frac{d \tilde{x}}{d t}=\tilde{u}-\tilde{c},
$$


in which the Riemann invariants $\tilde{k}_{ \pm}$are

$$
\begin{aligned}
& \tilde{\mathrm{u}}+2 \tilde{\mathrm{c}}-\tilde{\mathrm{m}} \mathrm{t}=\tilde{\mathrm{k}}_{+}=\text {constant along a curve } \tilde{\mathrm{C}}_{+}, \\
& \tilde{\mathrm{u}}-2 \tilde{\mathrm{c}}-\tilde{\mathrm{m}} \mathrm{t}=\tilde{\mathrm{k}}_{-}=\text {constant along a curve } \tilde{\mathrm{C}}_{-},
\end{aligned}
$$

where $\tilde{\mathbf{c}}=\sqrt{g \tilde{\mathrm{h}} \cos \theta}$ and $\tilde{\mathrm{m}}=-g \sin \theta+\tilde{\mathrm{F}}$.

\section{$3 \quad$ Existing solutions}

We review Ritter's solution to the dam break problem [8] and the solution due to Mangeney, Heinrich, and Roche for the debris avalanche problem [5]. Consider the dam break problem without friction on a horizontal bed in the standard Cartesian coordinate system given in Figure 1(a). This problem was solved analytically by Ritter [8] and Stoker [9]. Ritter's solution is

$$
u=\frac{2}{3}\left(\frac{x}{t}-c_{0}\right), \quad h=\frac{1}{9 g}\left(\frac{x}{t}+2 c_{0}\right)^{2}
$$

for $-2 c_{0} t \leqslant x \leqslant c_{0} t$, where $c_{0}=\sqrt{g h_{0}}$.

Mangeney, Heinrich, and Roche (MHR) [5] derived an analytical solution to the debris avalanche problem with friction in the topography-linked coordinate system, as shown in Figure 1(b). The MHR solution is

$$
\tilde{u}=\frac{2}{3}\left(\frac{\tilde{x}}{t}-\tilde{c}_{0}+\tilde{m} t\right), \quad \tilde{h}=\frac{1}{9 g \cos \theta}\left(\frac{\tilde{x}}{t}+2 \tilde{c}_{0}-\frac{1}{2} \tilde{m} t\right)^{2} .
$$

This solution is defined on the interval $-2 \tilde{\mathfrak{c}}_{0} t+\frac{1}{2} \tilde{\mathfrak{m}} t^{2}<\tilde{x}<\tilde{\mathfrak{c}}_{0} t+\frac{1}{2} \tilde{\mathfrak{m}} t^{2}$, where $\tilde{\mathrm{m}}=-\mathrm{g} \sin \theta+\tilde{\mathrm{F}}$ and $\tilde{\mathbf{c}}_{0}=\sqrt{\mathrm{g} \tilde{\mathrm{h}}_{0} \cos \theta}$. 


\section{A new solution}

This section derives an analytical solution to the debris avalanche problem in the standard Cartesian coordinate system using two methods, namely, the method of characteristics and a transformation technique.

Although the equations corresponding to the topography-linked coordinate system allow large bottom slopes, due to the initial condition used by Mangeney et al. [5], the MHR solution is accurate for mild slopes only. For steep slopes, soon after the wall given in Figure 1(b) is removed, some material around point $T_{1}$ would fall down and collapse with some material from around point $\mathrm{O}$ moving to the left. This supports the contention that the MHR solution is not accurate for steep slopes.

Here, we derive an analytical solution to a debris avalanche problem with a vertical wall on a sloping topography, as shown in Figure 1(c) in the standard Cartesian coordinate system. Our analytical solution is valid for small slopes only due to the Saint-Venant approach. However, the initial condition that we use involves a vertical wall, which is physically more realistic as it is more similar to some real world scenarios [1] than the problem considered by Mangeney et al. [5]. Our derivation utilises properties of the characteristics and follows closely the derivations of Mangeney et al. [5] and Stoker [9].

The debris avalanche (motion) at time $t>0$ consists of three zones: zone I is dry (debris free); zone II has a quadratic free surface; and zone III has a linear free surface. This is illustrated in Figure 2. After the wall is removed, the fluid in zone III moves with an acceleration $a$. To be consistent with the approximate nature of our basic equations (1) and (2) for shallow waves (consult the explanation of Dressler [3]), we take the horizontal acceleration $a=m$.

On the rightmost characteristic curve $\mathrm{C}_{+}$emanating from the origin, we have a velocity $u=a t$ and relative wave speed $c_{0}=\sqrt{g h_{0}}$. So, at any point $N$ on that curve, we have a velocity $u=a t_{N}$ and relative wave speed $c=c_{0}$ where 


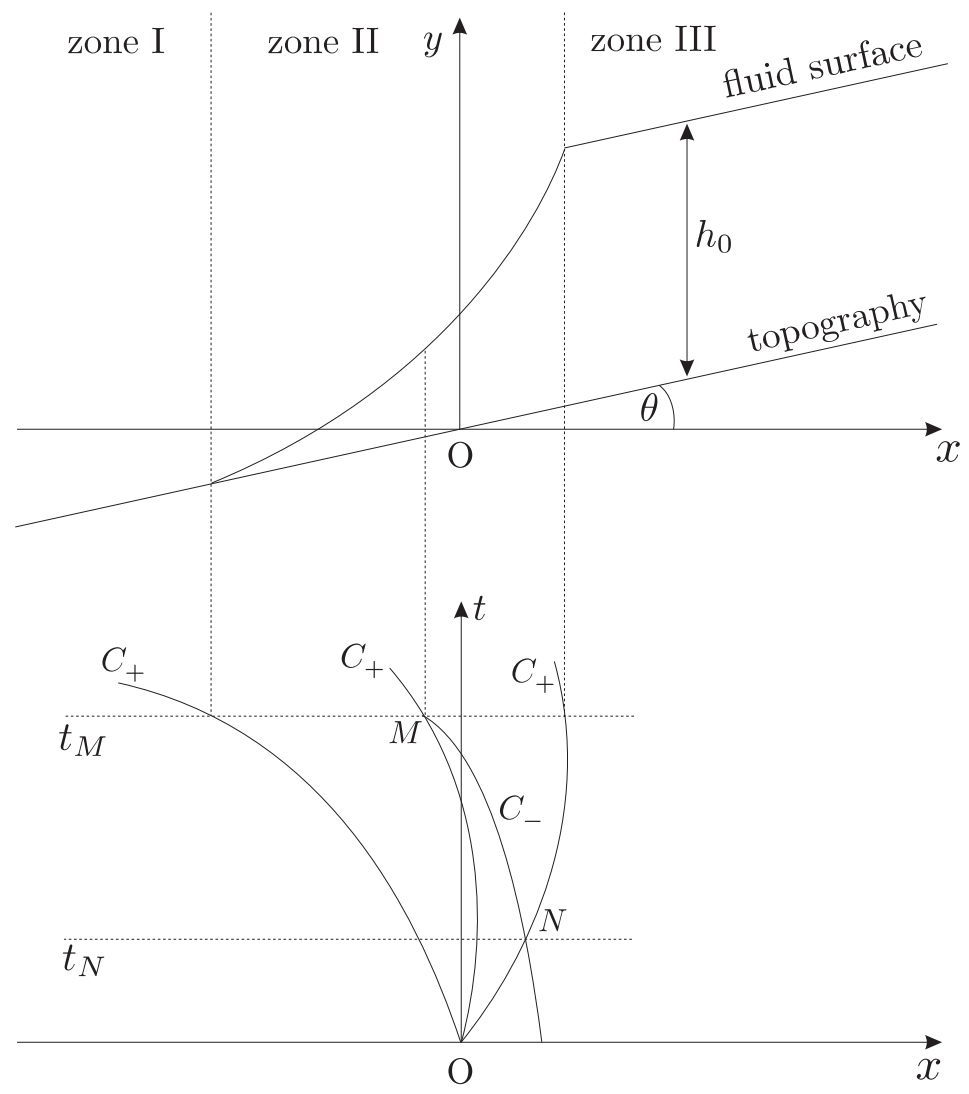

FiguRE 2: A debris avalanche and its characteristics.

$t_{N}$ is the time associated with point $N$. Now consider an arbitrary point $M$ in zone II such that $t_{M}>t_{N}$, where $t_{M}$ is the time associated with point $M$. Since $k_{-}$is constant along characteristic curve $C_{-}$passing through points $M$ and $\mathrm{N}$, and we have $\mathrm{a}=\mathrm{m}$, the velocity at point $M$ is

$$
u=2 c-2 c_{0}+m t,
$$

where $t_{M}$ is rewritten as $t$ for simplicity. The slope

$$
\frac{d x}{d t}=u+c=3 c-2 c_{0}+m t
$$


is the slope of each characteristic curve $C_{+}$in the rarefaction fan. Since $k_{+}$is constant along each curve defined by $d x / d t=u+c$ and since the velocity $u$ is given by (13), the relative wave speed $\mathbf{c}$ is constant along each curve in the rarefaction fan. As a result, equation (14) can be integrated to get

$$
c=\frac{1}{3}\left(\frac{x}{t}+2 c_{0}-\frac{1}{2} m t\right)
$$

that is,

$$
h=\frac{1}{9 g}\left(\frac{x}{t}+2 c_{0}-\frac{1}{2} m t\right)^{2} .
$$

Substituting (15) into (13), we obtain

$$
u=\frac{2}{3}\left(\frac{x}{t}-c_{0}+m t\right) .
$$

The fluid height must be nonnegative, so $c \geqslant 0$, and from (15) we get $x \geqslant-2 c_{0} t+\frac{1}{2} m t^{2}$. This implies that the tract of the front wave is $x=$ $-2 c_{0} t+\frac{1}{2} m t^{2}$. The tail wave follows the rightmost characteristic curve $C_{+}$ emanating from the origin, and satisfies $d x / d t=u+c=a t+c_{0}$. Integrating this differential equation and using $a=m$, we find that the tract of the tail wave is $x=c_{0} t+\frac{1}{2} m t^{2}$.

In summary, the analytical solution to the debris avalanche problem is (17) and (16) for $-2 c_{0} t+\frac{1}{2} m t^{2} \leqslant x \leqslant c_{0} t+\frac{1}{2} m t^{2}$.

Alternatively, we derive (17) and (16) using a transformation as follows. Substituting the new variables $[3,5]$

$$
\xi=x-\frac{1}{2} m t^{2}, \quad \tau=t, \quad v=u-m t \quad \text { and } \quad H=h
$$

into (1) and (2), we obtain

$$
\begin{aligned}
& \mathrm{H}_{\tau}+(v \mathrm{H})_{\xi}=0, \\
& (\mathrm{H} v)_{\tau}+\left(H v^{2}+\frac{1}{2} g \mathrm{H}^{2}\right)_{\xi}=0 .
\end{aligned}
$$


Therefore, for a given initial condition, if the solution to (19) and (20) is

$$
v=v(\xi, \tau) \text { and } \quad H=H(\xi, \tau),
$$

then the solution to (1) and (2) is

$$
u(x, t)=v(\xi, t)+m t \text { and } h(x, t)=H(\xi, t) .
$$

Employing this transformation together with Ritter's solution (11) given in Section 3, we get the solution (17) and (16).

\section{$5 \quad$ Numerical tests}

This section utilises our analytical solution to test a finite volume method (FVM) used to solve the Saint-Venant model.

Audusse et al. [2] established that in order to resolve the solution accurately at wet/dry interfaces, and to ensure that the FVM is well-balanced, stage $w:=h+z$ and height $h$ should be reconstructed. Therefore, here two methods with reconstruction based on stage $w$ and height $h$ are considered. We compare the performance of Method A (the FVM with reconstruction based on stage $w$, height $h$, and velocity $u$ ) to that of Method B (the FVM with reconstruction based on stage $\mathcal{w}$, height $h$, and momentum $p=h \mathfrak{c}$ ) in solving the debris avalanche problem.

Recall that Mangeney et al. [5] applied the Coulomb-type friction law

$$
\tilde{\mathrm{F}}=-\mathrm{g} \cos \theta \tan \delta \operatorname{sgn} \tilde{u},
$$

in which $\delta$ is a specified dynamic friction angle. We call $\tan \delta$ the friction slope. Following Mangeney et al. [5], we limit our discussion to the case when the friction slope is not larger than the bed slope, that is, $\tan \delta \leqslant \tan \theta$. This leads to $\operatorname{sgn}(u)=\operatorname{sgn}(\tilde{u})=-1$ for $t>0$. Then the horizontal component $F$ 
TABLE 1: Numerical settings in the simulations.

Description

Units of the measured quantities

Acceleration $g$ due to gravity

Minimum height allowed in the flux computation

Limiter for quantity reconstruction

Courant-Friedrichs-Lewy number

Error quantification

Spatial domain

Initial fluid height $h_{0}$ on the right of the wall
Specification

SI system

9.81

$10^{-6}$

minmod

1.0

discrete $\mathrm{L}^{1}$

$[-1000,1000]$

20

of $\tilde{\mathrm{F}}$, which is taken as a definition of the friction in the standard Cartesian coordinate system, is

$$
F=g \cos ^{2} \theta \tan \delta
$$

for $t>0$. Based on (23), at $t=0$, we have $F=0$ because of the zero velocity. In all simulations, the specification follows from our previous work [6], except for the initial fluid height $h_{0}$. We use the well-balanced finite volume scheme proposed by Audusse et al. [2] and extended by Noelle et al. [7] with the second order source, second order spatial, and second order temporal discretisations. Note that the discretisation of the friction term $h F$ included in the source at the $i$ th cell is $h_{i} F$, where $h_{i}$ is the value of the numerical height at the centroid of the cell and $F$ is as in (24). The central upwind flux formulation proposed by Kurganov, Noelle, and Petrova [4] is used to compute the numerical fluxes. Table 1 gives details of the numerical settings.

We assumed that $\tan \delta \leqslant \tan \theta$. Consequently, we have three possible test cases: $0=\tan \delta=\tan \theta ; 0=\tan \delta<\tan \theta$; and $0<\tan \delta \leqslant \tan \theta$. One representative of each case is considered. First, we test the numerical methods for a problem with friction slope $\tan \delta=0$ and bed slope $\tan \theta=0$. The errors for stage $w$ and momentum $p$ with various number of cells are presented in Table 2. Second, we consider a problem with friction slope $\tan \delta=0$ and bed slope $\tan \theta=0.1$. The errors for stage $w$ and momentum $p$ with various 


\begin{tabular}{|c|c|c|c|c|}
\hline \multirow{2}{*}{$\begin{array}{l}\text { Number } \\
\text { of cells }\end{array}$} & \multicolumn{2}{|c|}{$w$ error } & \multicolumn{2}{|c|}{$p$ error } \\
\hline & A & B & A & B \\
\hline 100 & 0.1074 & 0.1010 & 1.5234 & 1.4212 \\
\hline 200 & 0.0511 & 0.0499 & 0.7162 & 0.7067 \\
\hline 400 & 0.0254 & 0.0250 & 0.3537 & 0.3556 \\
\hline 800 & 0.0127 & 0.0125 & 0.1771 & 0.1785 \\
\hline 1600 & 0.0063 & 0.0063 & 0.0887 & 0.0894 \\
\hline
\end{tabular}

number of cells are presented in Table 3. Finally, for the third case we consider a problem with friction slope $\tan \delta=0.05$ and bed slope $\tan \theta=0.1$. The errors for stage $w$ and momentum $p$ with various number of cells are presented in Table 4. For this third case, Figure 3 shows the debris avalanche consisting of stage $w$ and momentum $p$ at time $t=15$ using Method B with 800 cells. Movies of the simulations using Method B are given in the supplementary files dam-break.avi ${ }^{1}$, avalanche-frictionless.avi ${ }^{2}$, and avalanche-friction.avi ${ }^{3}$ respectively for the first, second, and third case simulated with 800 cells for time $\mathrm{t} \in[0,15]$. Method A results in a similar figure and movies.

According to Tables 2-4, as the cell length is halved, the errors produced by the FVMs are halved. This suggests that we have only first order of convergence, even though we used second order methods. The reason is that a large error occurs around the front wave at the wet/dry interface; this is called the wet/dry interface problem. This problem was also identified in the simulations of Mangeney et al. [5]. Diffusion is also found around the tail wave, but it is not as significant as that around the wet/dry interface. Based on Tables 2-4, the error produced by Method B is slightly smaller than the

\footnotetext{
${ }^{1}$ http://anziamj . austms.org.au/ojs/index.php/ANZIAMJ/article/ downloadSuppFile/3785/697

${ }^{2}$ http://anziamj . austms.org.au/ojs/index.php/ANZIAMJ/article/ downloadSuppFile/3785/695

${ }^{3}$ http://anziamj . austms.org.au/ojs/index.php/ANZIAMJ/article/ downloadSuppFile/3785/696
} 


\begin{tabular}{|c|c|c|c|c|}
\hline \multirow{2}{*}{$\begin{array}{l}\text { Number } \\
\text { of cells }\end{array}$} & \multicolumn{2}{|c|}{$w$ error } & \multicolumn{2}{|c|}{$p$ error } \\
\hline & A & B & A & B \\
\hline 100 & 0.1121 & 0.1006 & 1.8559 & 1.5028 \\
\hline 200 & 0.0534 & 0.0501 & 0.8445 & 0.7428 \\
\hline 400 & 0.0257 & 0.0250 & 0.3885 & 0.3695 \\
\hline 800 & 0.0128 & 0.0126 & 0.1919 & 0.1846 \\
\hline 1600 & 0.0064 & 0.0063 & 0.0956 & 0.0925 \\
\hline
\end{tabular}

TABLE 4: Errors for $\tan \delta=0.05$ and $\tan \theta=0.1$ at $t=15$.

\begin{tabular}{c|cc|cc}
\hline Number & \multicolumn{2}{|c|}{$w$ error } & \multicolumn{2}{c}{$p$ error } \\
of cells & $\mathrm{A}$ & $\mathrm{B}$ & $\mathrm{A}$ & $\mathrm{B}$ \\
\hline 100 & 0.1092 & 0.1010 & 1.4858 & 1.2775 \\
200 & 0.0527 & 0.0504 & 0.6924 & 0.6402 \\
400 & 0.0256 & 0.0251 & 0.3285 & 0.3204 \\
800 & 0.0128 & 0.0126 & 0.1640 & 0.1611 \\
1600 & 0.0064 & 0.0063 & 0.0823 & 0.0810 \\
\hline
\end{tabular}

error produced by Method A in solving the debris avalanche problem.

\section{Conclusions}

We used a Saint-Venant approach in the standard Cartesian coordinate system to solve the debris avalanche problem. The drawback of this approach is that the solution is not physically valid for a very steep sloping topography. Future research is to seek a better approach to solve the debris avalanche problem analytically and to find a numerical technique that can be used to resolve the wet/dry interfaces accurately. Our analytical solution might help in developing a numerical scheme to resolve the solution at these interfaces. 

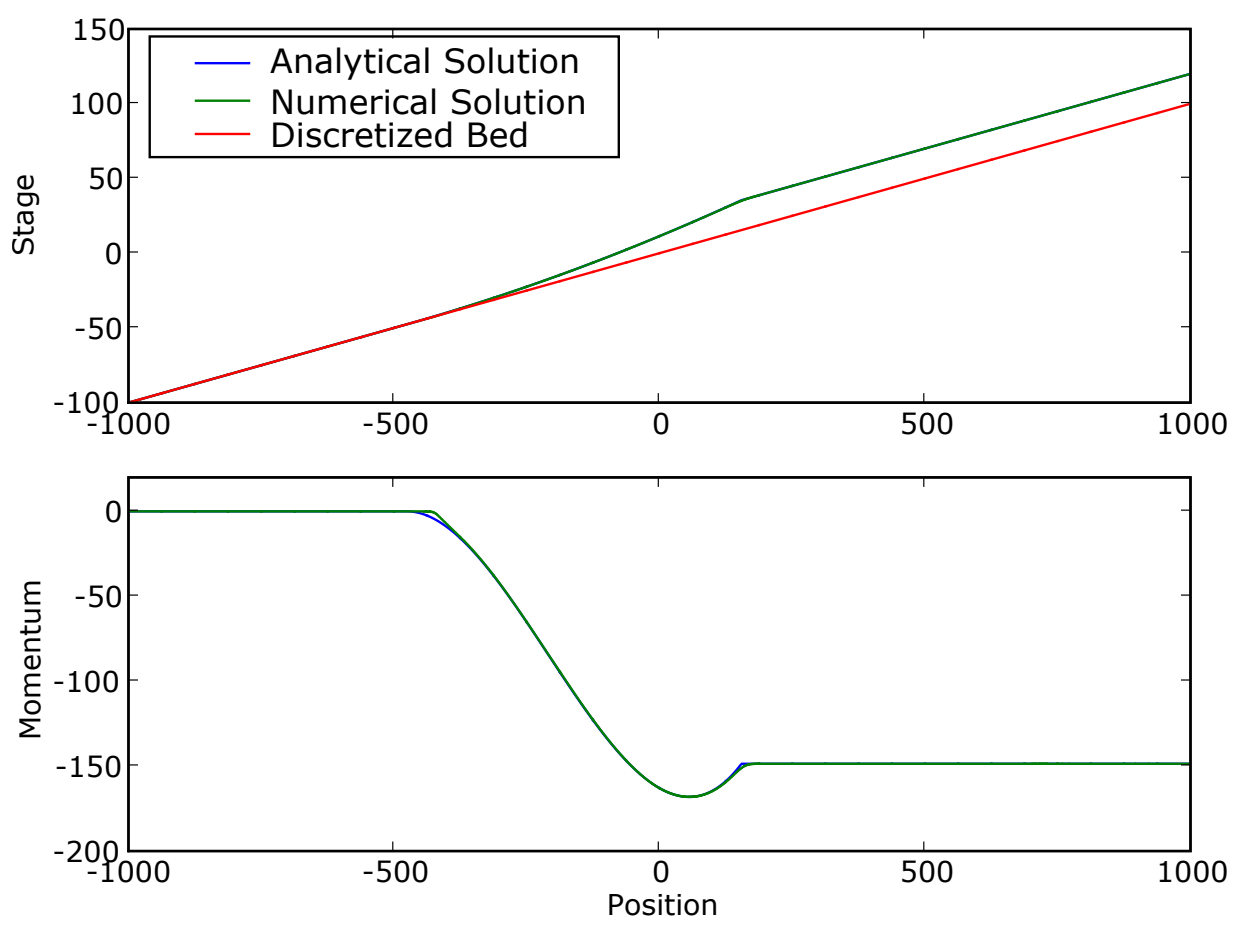

Figure 3: Avalanche with $\tan \delta=0.05$ and $\tan \theta=0.1$ at $\mathrm{t}=15$.

Acknowledgements The work of Sudi Mungkasi was supported by ANU $\mathrm{PhD}$ and ANU Tuition Scholarships. We thank Christopher Zoppou for stimulating discussions.

\section{References}

[1] C. Ancey, R. M. Iverson, M. Rentschler, and R. P. Denlinger. An exact solution for ideal dam-break floods on steep slopes. Water Resour. Res., 44(1):W01430 pages 1-10, 2008. doi:10.1029/2007WR006353 C354 
[2] E. Audusse, F. Bouchut, M. O. Bristeau, R. Klein, and B. Perthame. A fast and stable well-balanced scheme with hydrostatic reconstruction for shallow water flows. SIAM J. Sci. Comput., 25(6):2050-2065, 2004. doi:10.1137/S1064827503431090 C357, C358

[3] R. F. Dressler. Unsteady non-linear waves in sloping channels. Proc. Royal Soc. London, Ser. A, 247(1249):186-198, 1958. doi:10.1098/rspa.1958.0177 C354, C356

[4] A. Kurganov, S. Noelle, and G. Petrova. Semidiscrete central-upwind schemes for hyperbolic conservation laws and Hamilton-Jacobi equations. SIAM J. Sci. Comput., 23(3):707-740, 2001. doi:10.1137/S1064827500373413 C358

[5] A. Mangeney, P. Heinrich, and R. Roche. Analytical solution for testing debris avalanche numerical models. Pure Appl. Geophys., 157(6-8):1081-1096, 2000. doi:10.1007/s000240050018 C350, C353, C354, C356, C357, C359

[6] S. Mungkasi, and S. G. Roberts. On the best quantity reconstructions for a well balanced finite volume method used to solve the shallow water wave equations with a wet/dry interface. ANZIAM J., 51(EMAC2009):C48-C65, 2010. http://anziamj . austms.org.au/ ojs/index.php/ANZIAMJ/article/view/2576 C358

[7] S. Noelle, N. Pankratz, G. Puppo, and J. R. Natvig. Well-balanced finite volume schemes of arbitrary order of accuracy for shallow water flows. J. Comput. Phys., 213(2):474-499, 2006. doi:10.1016/j.jcp.2005.08.019 C358

[8] A. Ritter. Die fortpflanzung der wasserwellen. Zeitschrift des Vereines Deutscher Ingenieure, 36(33):947-954, 1892. C350, C353

[9] J. J. Stoker. Water Waves: The Mathematical Theory with Application. Interscience Publishers, New York, 1957. C352, C353, C354 


\section{Author addresses}

1. Sudi Mungkasi, Mathematical Sciences Institute, The Australian National University, Canberra, Australia; and Department of Mathematics, Sanata Dharma University, Yogyakarta, InDONESIA. mailto: sudi.mungkasi@anu.edu.au, sudi@usd.ac.id

2. Stephen G. Roberts, Mathematical Sciences Institute, The Australian National University, Canberra, Australia. mailto:stephen.roberts@anu.edu.au 